\title{
Evidence of the Relationship between the Emerging Magnetic Fields, Electric Currents, and Solar Flares Observed on May 10, 2012
}

\author{
M. A. Livshits ${ }^{1 *}$, \\ I. Yu. Grigoryeva², \\ I. I. Myshyakov ${ }^{3}$, and \\ G. V. Rudenko ${ }^{3}$
}

\begin{abstract}
We have analyzed multi-wavelength observations and magnetic-field data for the solar flare of May 10, 2012 (04:18 UT) and have detected a sign inversion of the signal in the line-ofsight magnetic measurements in the umbra of a small spot. This effect is associated, at least partly, with the emergence of a new magnetic field. Almost at the same time, a burst of hard X-rays was recorded, and a "sunquake" was generated due to the impact of the disturbance in the energy release range on the photosphere. At the beginning of the event, a sigmoid flare was recorded, but it did not spread, as it usually does, along the polarity inversion (neutral) line. SDO/HMI full-vector measurements were used to extrapolate the AR 11476 magnetic field to the corona, and the distribution of vertical currents $j_{z}$ in the photosphere was obtained. The distribution of currents in the active region shows that the relationship between them and the occurrence of flares is very intricate. We have corroborated that the expected "ideal" behavior of the current system before and after the flare (e.g., see (Sharykin and Kosovichev, 2015)) is observed only in the sigmoid region. The results obtained were compared with the observations of two other flares recorded in this AR on the same day, one of which was similar to the flare under discussion and the other was of different type. Our results confirm that the formation and eruption of large-scale magnetic flux ropes in sigmoid flares are associated with the shear motions in the photosphere and the emergence of twisted magnetic tubes, as well as with the subsequent development of the torus instability.
\end{abstract}

\section{Keywords}

Sun: flares, X-ray, radio, sunquake, magnetic fields, currents, torus instability

${ }^{1}$ Pushkov Institute of Terrestrial Magnetism, Ionosphere and Radio Wave Propagation of Russian Academy of Sciences, Moscow, Troitsk, Russia

${ }^{2}$ Main Astronomical (Pulkovo) Observatory, Russian Academy of Sciences, Pulkovo, St. Petersburg, Russia

${ }^{3}$ Institute of Solar-Terrestrial Physics, Siberian Branch, Russian Academy of Sciences, Irkutsk, Russia

* Corresponding author: maliv@mail.ru 


\section{Contents}

1 Introduction

2 Electromagnetic Emission in the Flare of May 10, 2012 (04:18 UT)

3 Emergence of Magnetic Fields

4 Extrapolation of Fields to the Corona and Distribution of Electric Currents in AR 11476

5 The Other Flares of May 10, 2012

6 Results and Discussion

References

\section{Introduction}

Over the past 15 years of the new century, TRACE, RHESSI, CORONAS-F, and other spacecrafts have provided a wealth of data on nonstationary processes in the Sun. The characteristics of X-ray sources and the relationship with the microwave and optical emission of solar flares have been studied in detail (Krucker et al., 2008). In the past 50 years it was believed that these events occurred in the corona and were due to reconnection of the magnetic field lines. However, the recent Hinode, SDO/HMI, and AIA full-vector magnetic observations (Schou, et al., 2012; Lemen, et al., 2012) and the theoretical extrapolation of photospheric magnetic fields to the corona (Metcalf, et al., 2008) have shown that, in the course of evolution of active regions, the magnetic-field energy is accumulated at very low altitudes in the chromospheres, where the Lorentz forces operate, and electric currents are amplified significantly. The free energy of the currents is released at low altitudes giving rise to flares, coronal mass ejections (CME), and "sunquakes". This makes us turn from the recently introduced term "coronal flare" back to the notion of chromospheric flares, which was widespread in the 1960-1970-ies. This opinion is supported even by the advocates of the reconnection theory as a mechanism of flares (Fletcher et al., 2011).

At the early stage of measurements of solar magnetic fields, A.B.Severny (1988) showed that the sources of individual flare nodes in the chromosphere are located in the vicinity of the polarity inversion line. He and his co-authors showed also that these nodes arise in the areas of a high-magnetic field gradient. The first full-vector measurements of AR magnetic fields allowed them to study the relationship between the electric currents and the process of evolution of solar flares. However, the correlation between the distribution of the vertical magnetic field and the flare nodes in the chromospheric $\mathrm{H}$-alpha line proved to be very complicated. Only now the problem discussed by A.B.Severny and D.Rust (Rust, 1968) can be investigated more or less comprehensively using modern observations of the magnetic-field dynamics, multi-wavelength observations of flares, and new theoretical concepts of electromagnetic processes in the Sun.

Recent studies of the relation between the evolution of magnetic fields and the nonstationary processes are based on several earlier results. An important finding was the emergence of new magnetic fields. The change in the field configuration can result in a sudden release of energy or can intensify the emission of impulsive flares along with the generation of plasma motions (CME etc.) and EUV waves (sunquakes). The heating of plasma in the chromosphere may be due to the dissipation of currents (Zaitsev and Stepanov, 2008), to the Lorenz forces (Fisher, et al., 2012), or to the energy loss of accelerated particles at the beginning of the gas-dynamic response considered first by Kostyuk and Pikelner (1975). To understand the origin of nonstationary processes, it is necessary to note that in some cases before or during the flare a large-scale flux rope emerges usually in the vicinity of the polarity inversion line lead- 


\section{Evidence of the Relationship between the Emerging Magnetic Fields, Electric Currents, and Solar Flares Observed on May 10, 2012 - 3/16}

ing to the occurrence of a sigmoid flare (see (Hood et al., 2012, and references therein) and to subsequent formation of post-eruption arcades.

Thus, the appearance of new theoretical and observational data required new concepts of the development of MHD processes in the outer atmosphere of the Sun. These concepts should take into account the direct effect of currents on the heating and motion of plasmas in the chromosphere and its behavior in the force-free and potential fields in the corona, including the possibility of reconnection of the field lines and formation of thin current sheets. Of primary importance in approaching this problem as a whole is the simulation of the AR current system. In doing so, we must take into account the topology of magnetic fields in the AR or in the complexes of activity, which was studied first by Lee et al. (2010). A sudden reconstruction of the magnetic configuration proves to be closely related to the development of nonstationary phenomena. The role of shear motions in such reconstruction was considered earlier (Matyukhin and Tomozov, 1991, see following discussion in Prist and Forbes, 2000) usually for the processes with reconnection in the corona. According to the numerical model by Hood et al. (2012), the emergence of twisted flux ropes is indicative of such reconstruction of the magnetic configuration and current system.

Of course, it is important to find observational evidence that would support or disprove the new theoretical conjectures. Perhaps one of the first publications in this context was the work by Sharykin and Kosovichev (2015), where Figure 10 represents schematically a current system with vertical currents $j_{z}$ of opposite signs on either side of the polarity inversion line. Indeed, such a picture would be expected in the simplest case of transition from the twisted flux rope to the flare post-eruption phase. Now, however, there are numerous full-vector measurements in many active regions where the distribution of the vertical current $j_{z}$ is restored. As in the pioneering work by Severny, this distribution throughout the AR does not correspond to the ideal pattern.

In this work we made an attempt to investigate the relationship between the currents and the development of non-stationary processes on the example of the events on 2012 May 10. The first flare with the maximum at 04:18 UT (GOES) was unusual since the emergence of the new field in it was observed in the umbra of the small spot with the simultaneous hard X-ray burst. This event was accompanied by a sunquake. The second flare with the maximum at 05:10 UT was more typical, and the third flare (20:26 UT) was like the first one, but less powerful. After the Introduction, in Section 2 we analyse the emission of the first flare. In Section 3 the analysis of the observations of the magnetic field emergence at 04:00 UT is given, in Section 4 the behavior of the photospheric and coronal magnetic fields is discussed, and in Section 5 the data are briefly compared with that on the two subsequent flares. In the last section we discuss the question of the origin and the possible model of sigmoid flares with the ejection of large twisted magnetic ropes.

\section{Electromagnetic Emission in the Flare of May 10, 2012 (04:18 UT)}

AR 11476 appeared on the disk on May 5, 2012, and by May 10, its area reached 1000 m.v.h. The corresponding sunspot group consisted of a large, complex leading spot and minor spots at the center and in the tail part of the group. On the day under discussion, the AR produced two large M-flares and a series of weak flares of class C. Here, we consider the first flare M5.7 (04:11-4:23, maximum at 4:18 UT, GOES) and then compare it with the T7.9 flares that occurred at the decay of the first one and with the M1.7 flare observed in the same AR in the evening of that day (see Fig. 1).

The first flare was ordinary as to its X-ray and microwave emission. It occurred in close proximity to a small spot at the center of the group near the polarity inversion line, had impulsive nature, and was rather hard.

The left-hand part of Fig. 2 represents the entire AR 11476 in the white light (SDO/HMI_Ic). The 


\section{Evidence of the Relationship between the Emerging Magnetic Fields, Electric Currents, and Solar Flares Observed on May 10, 2012 - 4/16}
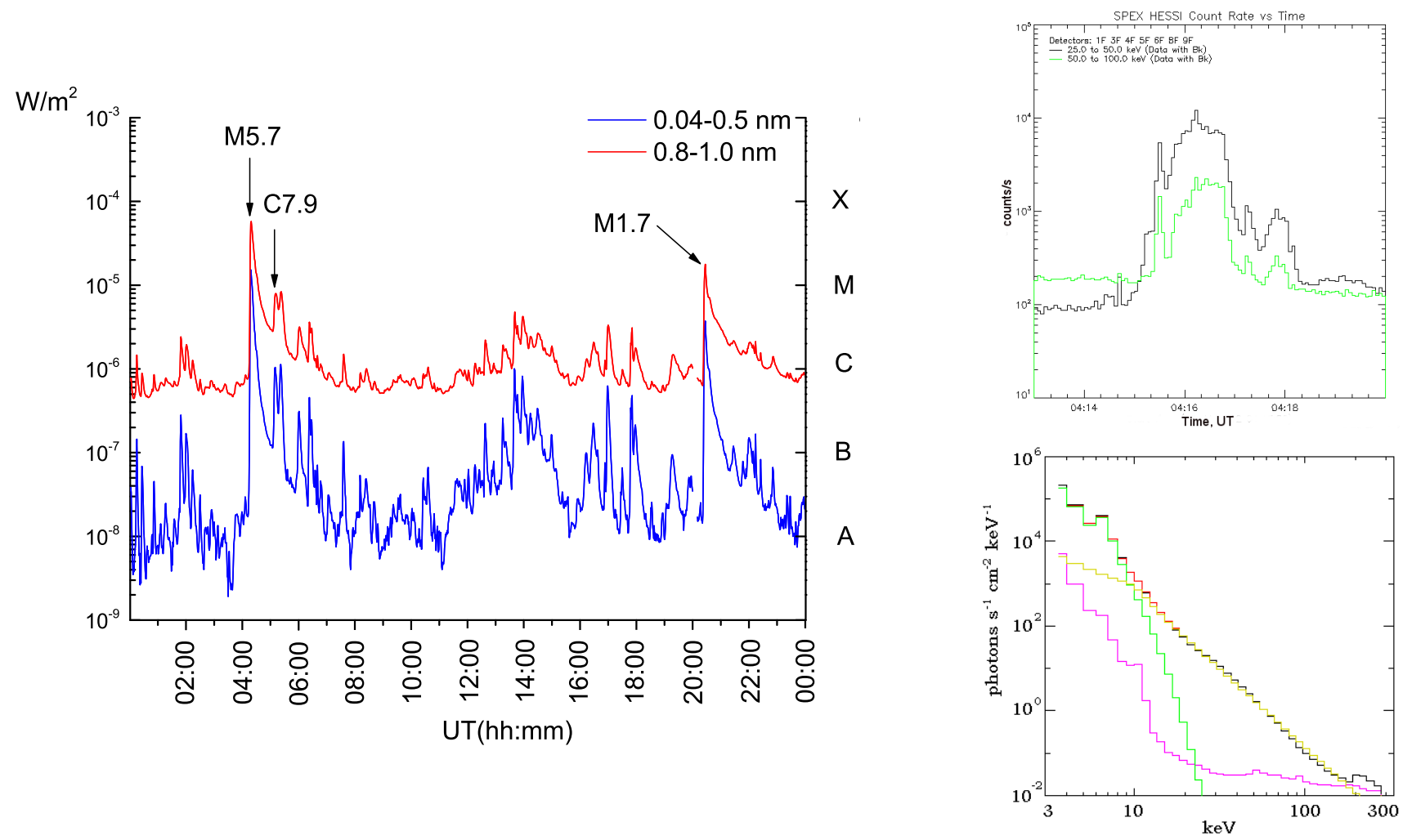

Figure 1. Left: diurnal GOES SXR time profiles (the lines show the flares under examination). Right, at the top: the RHESSI HXR time profile- count rates above the background for the photon energy in the range $25-50 \mathrm{keV}$ (black line) and 50-100 keV (green line), at the bottom - the flare spectrum at 04:15:44 UT on May 10, 2012 where the background is shown as magenda line. The flare emission is divided into the thermal (green line) and nonthermal (orange line) components.

rectangle on the right shows region (A), where the flare was observed, and the squared region (B) is where a change of sign of the signal was recorded on the line-of-sight magnetogram (SDO/HMI_LOS). The same flare in the $131 \AA$ line (SDO/AIA) at the growth of its intensity is also shown on the right.

In general, judging by its X-ray emission recorded by GOES and RHESSI (Lin, et al., 2002), this was a typical event somewhat harder than an average flare of importance M5 as seen from Fig. 1 (the tilt of the photon spectrum $\gamma \approx 3.0$ in the energy range of $20-80 \mathrm{keV}$, Fig. 1).

The microwave flux was also characteristic of the flares of this class with a complex behavior of the polarized component (Stokes parameter V). The time profiles of intensity (Stokes parameter I) and polarization (Stokes parameter V) at the frequencies of 17.0, 9.4, 3.75 and 2.0 GHz based on the Nobeyama polarimeter data (NoRP: Shibasaki et al., 1979; Nakajima et al., 1985) are given on the left and at the center of Fig. 3. The values and the dynamics of fluxes correspond to the majority of flares of this intensity. As usual, there are two discernible footpoints of the flare loop, located in the magnetic fields of the opposite signs (Fig. 3, right).

On the other hand, the polarization demonstrates a rather complicated time behavior. In particular, In particular, we have to be an inversion of the polarized emission sign at the frequency of $9.4 \mathrm{GHz}$. Besides, a sudden change of sign is observed at $2 \mathrm{GHz}$ at 04:16 UT. Such a picture of evolution of the polarized microwave emission may be associated with a complex AR topology in the lower corona. 


\section{Evidence of the Relationship between the Emerging Magnetic Fields, Electric Currents, and \\ Solar Flares Observed on May 10, 2012 - 5/16}
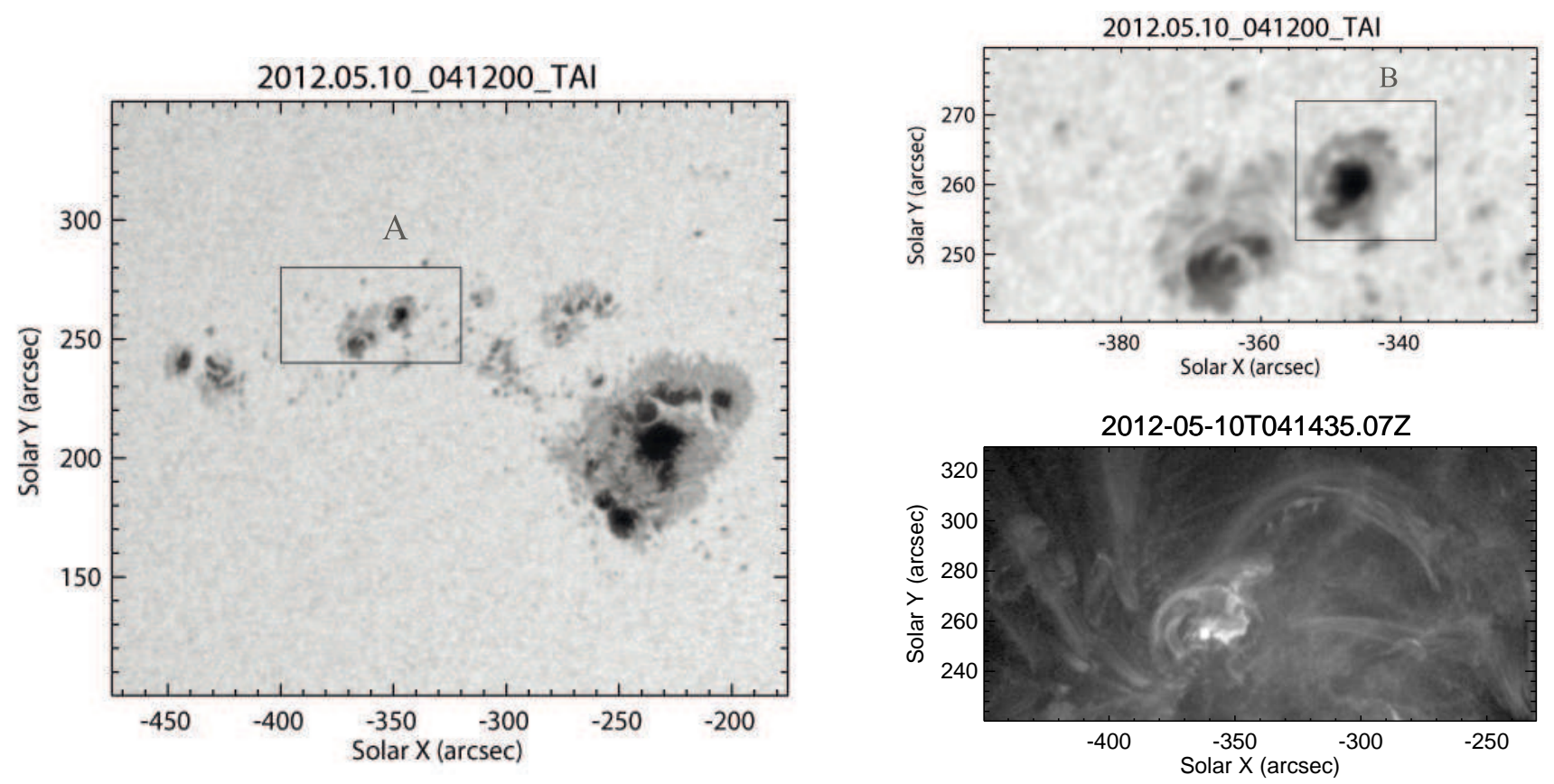

Figure 2. Left: the sunspot group in AR 11476 at 04:12 UT (SDO/HMI_Ic), the rectangle is the selected region A. Right: region A with the square region B for the same time (at the top); flare in the $131 \AA$ line (SDO/AIA) in the vicinity of the maximum (at the bottom). The scales show the distance from the disk center in arcsec.
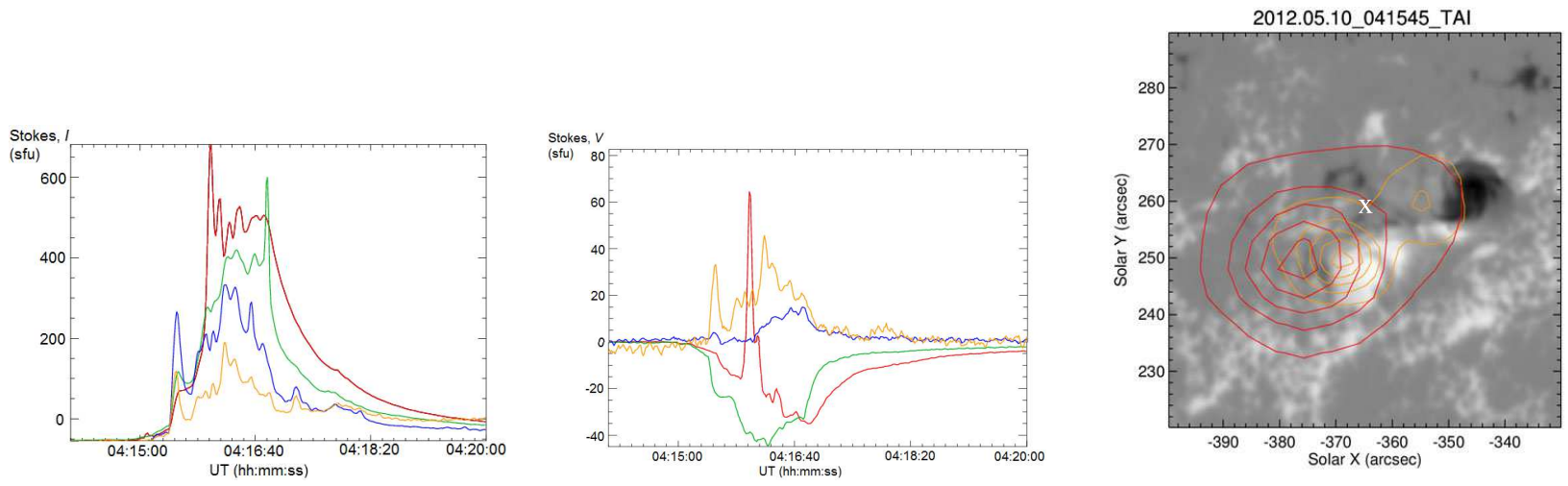

Figure 3. Evolution of the microwave flux from the M5.7 flare on May 10, 2012 (Stokes parameters I (left) and $\mathrm{V}$ (in the centre) at the frequencies of $2 \mathrm{GHz}$ (red) $3.75 \mathrm{GHz}$ (green), 9.4 GHz (blue) and $17 \mathrm{GHz}$ (yellow) (Nobeyama, NoRP). Right - a fragment of the LOS-magnetogram of the flare generation region in the phase of maximum: the scales provide the distance from the disk center in arcsec; the contours correspond to the emission at $34.0 \mathrm{GHz}$ (yellow line) and 17.0 GHz (red line) (Nobeyama, NoRH) at 04:15:30 UT; these isophotes correspond $90 \%, 70 \%, 50 \%, 30 \%$ and $10 \%$ of the maximum at $17 \mathrm{GHz}$ and $34 \mathrm{GHz}$. The sign $\mathrm{X}$ shows the flare occurrence site according to HXR RHESSI data. 


\section{Evidence of the Relationship between the Emerging Magnetic Fields, Electric Currents, and Solar Flares Observed on May 10, 2012 - 6/16}

\section{Emergence of Magnetic Fields}

It is well known that in many cases, a relationship can be observed between the occurrence of a flare and the emergence of a new magnetic field. However these two processes do not usually coincide in time and space. In other words, the emergence of a new magnetic field violates the stability of the magnetic configuration. The flare in a given AR may occur far enough from the emerging field, before or after the emergence or, even between its separate episodes. In the case under consideration, the two processes coincided in space and time. Besides that, though usually the new magnetic field appears in the vicinity of the polarity inversion line separating the fields of medium-high intensity, in our case, the emergence occurred in the umbra of a small spot at the AR center (square B in Fig. 2). Here, the change of sign of the signal (SDO/HMI_LOS) is indicative of the field emergence, but still it certainly does not mean the inversion of the field sign.

We have analyzed the Stokes parameters recorded in the supposed emergence area and obtained the Gaussian distribution. Of course, strong plasma streams may also interfere with the field measurements. One of the arguments for the reality of the field emergence is the fact that a similar picture was observed during the flare recorded at the same site in the same AR later in the evening at 20:26 UT.

The evolution of the emerging field in region $\mathrm{B}$ at some selected points of time based on the SDO/HMI_LOS magnetograms is illustrated in Fig. 4. The reversed sign of the signal was observed during 4 min., after which the signal was restored to its former value during another 4 min. Unfortunately, full-vector magnetic data are only available for the moments before and after the emergence. Note that the upward directed motions are registered according to the data of SDO/HMI in the frame 04:16:30 UT.

During the event under discussion, a sunquake was recorded in the vicinity of the hard X-ray maximum (Buitrago-Cass, et al., 2015). Such a response to the sudden energy release in the photosphere is projected onto the point at the peak of a low flare loop. The asterisk (X) in the right-hand part of Fig. 3 marks the position of the hard X-ray source at the maximum of the burst. The isolines of radio brightness at 17.0 and $34.0 \mathrm{GHz}$ superimposed on the line-of-sight distribution of magnetic fields at the maximum of the event are given according to the Nobeyama radio heliograph data (NoRH: Nakajima et al., 1994). The footpoints of the low flare loop seen in different SDO/AIA ranges are best pronounced at the high frequency of $34.0 \mathrm{GHZ}$. In this case, the sunquake is most likely bound to the time and site of the primary energy release. This suggestion agrees with the sunquake data available in (Sharykin and Kosovichev, 2015; Sharykin, Kosovichev, and Zimovets, 2015)

\section{Extrapolation of Fields to the Corona and Distribution of Electric Currents in AR 11476}

In recent years, our understanding of the role of magnetic fields in the development of activity in the outer atmosphere of the Sun has changed. It was found that the magnetic beta (the ratio of the gas pressure to the magnetic pressure), which is close to unity immediately above the photosphere, becomes very small in the chromosphere and corona. As a result, the plasma at the base of the active region is exposed to the Lorenz forces, while higher the field becomes force-free. At the same time, the evolution of fields in the chromosphere can lead to a significant excess of the magnetic-field energy over the corresponding value of the force-free (potential) field, and this free energy can be spent on the development of nonstationary processes.

Full-vector magnetic observations in the photosphere allow us to extrapolate the nonlinear force-free fields up to the corona. This method called NLFFF (nonlinear force-free field) extrapolation is widely used in heliophysics (e.g., see (Metcalf et al, 2008)). 

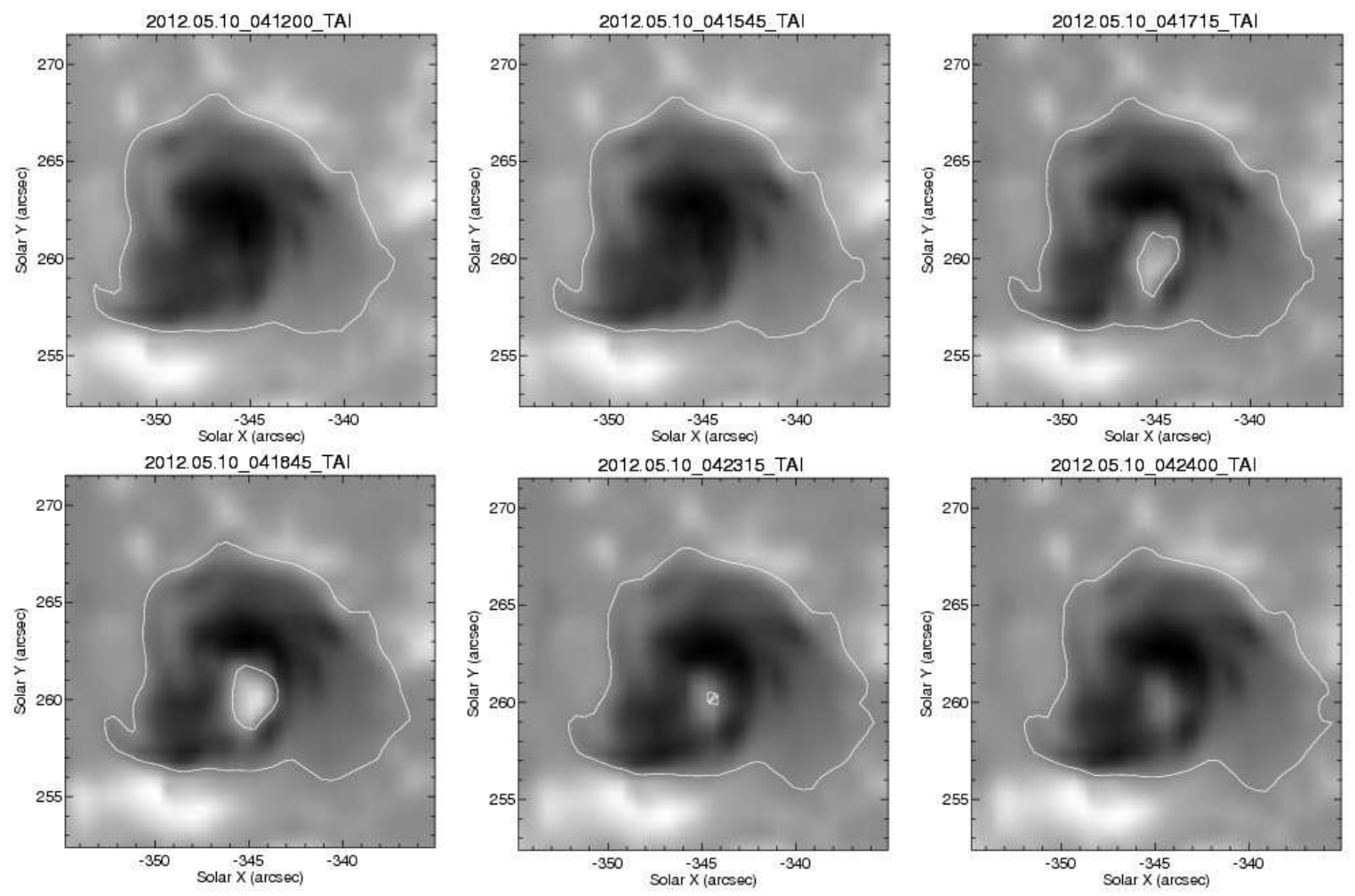

Figure 4. Fragments of the image of the magnetic-field line-of-sight component in the emergence region in AR NOAA 11476 (SDO/HMI_LOS) on May 10, 2012 at the selected moments during the flare. The contours show the isolated local magnetic field along the polarity inversion line. The scales provide the distance from the center of the disk in arcsec. 


\section{Evidence of the Relationship between the Emerging Magnetic Fields, Electric Currents, and Solar Flares Observed on May 10, 2012 - 8/16}

In our work, for the calculation of the coronal magnetic field in the non-linear force-free approximation an optimisation method (first proposed in (Wheatland et al, 2000)) is employed in the implementation of (Rudenko and Myshyakov, 2009). The core of the method is the subsequent transformation of some initial (potential) distribution of the magnetic field toward the force-free structure in accordance with the photospheric magnetogram. To do this a functional of the following type is introduced:

We have performed calculations based on the solution of the boundary-(Rudenko and Myshyakov, 2009) value problem for the nonlinear force-free field using minimization of the functional introduced in:

$$
L=\int_{V}\left[B^{-2}|[\nabla \times \mathbf{B}] \times \mathbf{B}|^{2}+|\nabla \cdot \mathbf{B}|^{2}\right] d V
$$

which equals 0 if the field $B$ is force-free and is positive in the opposite case.

Thus, in the course of the solution of the functional (1) minimisation problem, the field in the volume $V$ acquires the force-free configuration. As the initial distribution we use a potential field calculated from the normal component of the photospheric field via fast Fourier transform (FFT). A remarkable feature of the optimisation method's realisation, which we apply, is the use of the full system of the evolution equations of the field (see (Rudenko and Myshyakov, 2009)).

The field lines in region A shown in Fig. 2 were calculated up to the height of $25000 \mathrm{~km}$. The result is represented in Fig. 5. One can see that at the very beginning of the flare, the force lines around the field hill in a small spot are clustering along the polarity inversion line, and the flare nodes are formed in the region of their highest concentration (see the EUV background on the negative image in Fig. 5). Generally speaking, a bunch of force lines covering a significant part of the polarity inversion line, particularly, where it separates the hills of strong magnetic fields, can form in many flares. In our case, it can be supposed that a small bunch was formed only on the south side of a small spot at the center of the group. As in other sigmoid flares, such a shape of the field lines in AR stimulates the development of sigmoid flare. In this case, the field reconstruction occurred almost immediately after the formation of currents over the neutral line.

Figure 5 shows only closed field lines in the selected region A in projection on the plane of the sky. We can see a small spot of negative polarity fringed with a bunch of field lines that continue further beyond the neutral line. At present it is believed that this is where the main force-free currents usually identified with the plasma flux rope are flowing. It is important to note that this bunch of field lines coincides with the position of the flare nodes forming a small sigmoid.

As shown by NLFFF-extrapolation, there are also field lines rising up to $5000 \mathrm{~km}$ and higher and connecting the small spot of negative polarity with the hill of the field of opposite sign. They are located above the bunch of field lines and perhaps prevent the ejection. In the course of the flare evolution as inferred from SDO/AIA EUV data, the flare loops 10-20 thousand km high were formed, but plasma did not escape into interplanetary space (i.e., a CME did not occur as it follows from the radio data: there is no a type II burst etc.).

Then at the photosphere level we have calculated the distribution of vertical currents $j_{z}$ throughout the AR on May 10, 2012 for the time interval from 03:48 UT to 05:48 UT using the full-vector magnetic data with a step of $720 \mathrm{~s}$. The analysis of the location of flares on that day corroborates the correlation between the sites of occurrence of the flare nodes and the areas of enhanced current (Grigor'eva et al., 2013). However, no definite regularity was revealed in the behavior of the currents throughout the AR and in the occurrence of flares. Namely, all currents are concentrated along the neutral line, including the area near spots. Therefore, the changes that may occur in the middle of the neutral line in AR are not identified in the total current of one and the other sign. Sharykin and Kosovichev (2015) drew attention 


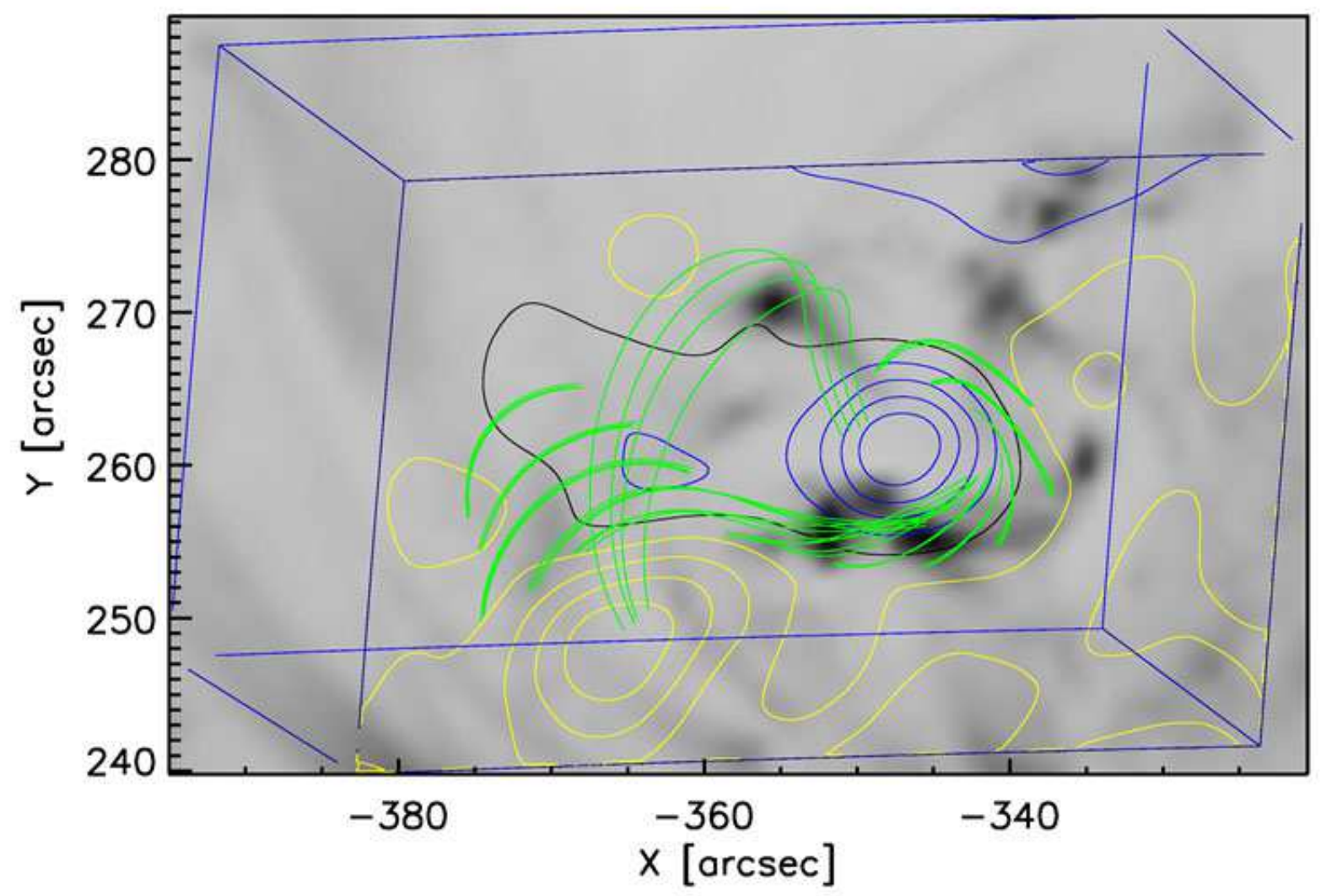

Figure 5. The background is a fragment of the inverted image of AR NOAA 11476 (SDO/AIA) in the EUV line $171 \AA$ Abtained on May 10, 2012 at 04:12:00 UT. The contour lines show the line-of-sight components of the magnetic field of positive (white) and negative (black) sign in the flare region at $80 \%, 60 \%, 40 \%$, and $20 \%$ of the maximum at the same time. The white line presents force lines above the neutral line and the higher loops connecting hills of the magnetic field of the opposite signs. The direct blue lines show the projection cube with the height of $25000 \mathrm{~km}$. The scales provide the distance from the center of the disk in arcsec. 


\section{Evidence of the Relationship between the Emerging Magnetic Fields, Electric Currents, and Solar Flares Observed on May 10, 2012 - 10/16}

to the fact that in an "ideal" current system, the mean currents of each sign must increase on either side of the polarity inversion line. In our case, like in the studies of the 1970-ies considering the relationship between the vertical currents and flares, this effect is very weak in respect to the entire AR.

Since in the growth phase of the flare the largest changes in the magnetic field are recorded at the site of occurrence of the sigmoid, we have studied this effect in the selected region A (see Fig. 1). Figure 6 illustrates the distribution of the vertical currents in the photosphere for two points of time. One can see that the currents of different sign contact at the point $Y 256, X-350$ arcsec. This area coincides with the region of the field lines concentration in Fig. 5 above the point on the polarity inversion line in the region of strong fields (see Fig. 4).

Generally, the evolution of the currents pattern is traced upon the set of the $j_{z}$ maps constructed on the basis of the data on the full vector of the field. The largest change of the currents takes place in the pointed region of the sigmoid localization. The subsequent evolution of the current system appears to be tightly bound with the development of numerous weak flares in AR 11476. Note also that the substantial change of the currents took place at 04:00-04:24 UT in the area $Y 250, X-364$ arcsec which is the footpoint of the loop connecting the place of the magnetic field emergence with the hill of the positive polarity (see Fig. 5).

Except the change of the currents pattern, the difference between two images on left part of Fig. 6 is manifested in all the current values, the fact which in this example is revealed even in the difference of the scales.

To study this effect, we have determined the mean value of the vertical current in the whole region (A). The averaging was done separately for the pixels with the positive and negative fields. In the time interval from 04:00 UT to 05:48 UT in Fig. 6 one can observe the general trend of the averaged vertical current $j_{z}$ of both signs. The whole trend represents the fact that the flaring activity of this AR on that day was gradually increasing.

The effect related to the flare in question is manifested as the increase of the averaged currents during the flare 04:00 UT-04:24 UT. According to the hard X-ray data, the maximum of the flare falls on 04:16:30 UT (see Fig. 1). In our case the maximum value corresponds to the time 04:24 UT and falls down afterwards. Such character of the change of $j_{z}$ is noticed in (Sharykin and Kosovichev, 2015) as well. The maximum value is small and only slightly exceeds the value of $3 \sigma$ (its $\sigma$ value of approximately $2.3 \cdot 10^{-4} \mathrm{~A} / \mathrm{m}^{2}$ is determined from the change of the currents in the area of the same active region in the quiescent state).

Note also that the flare maximum pointed out occured between 04:12 UT and 04:24 UT. Unfortunately, there is no freely accessed data on the full magnetic field vector with better time resolution, therefore our effect of the change of the currents' characteristics during this flare can be somewhat underestimated.

\section{The Other Flares of May 10, 2012}

AR 11476, which we are discussing, displayed a high flare activity. Every day since May 6 it produced a large number of weak C-flares and, on some days until May 17, 1 or 2 M-flares.

In addition to the flare of importance M5.7 we are considering here, it produced on the same day 16 C-flares and one flare of importance M1.7 recorded at 20:20 UT. A change in the signal intensity was recorded in the umbra of the same spot as in the first M-flare according to the line-of-sight field distribution (SDO/HMI_LOS). The duration of this effect was two times less than in the first flare (from 20:24 UT to 20:29 UT). However here, unlike the M5.7 flare, the signal on the HMI_LOS magnetogram in the emergence region did not change the sign. 

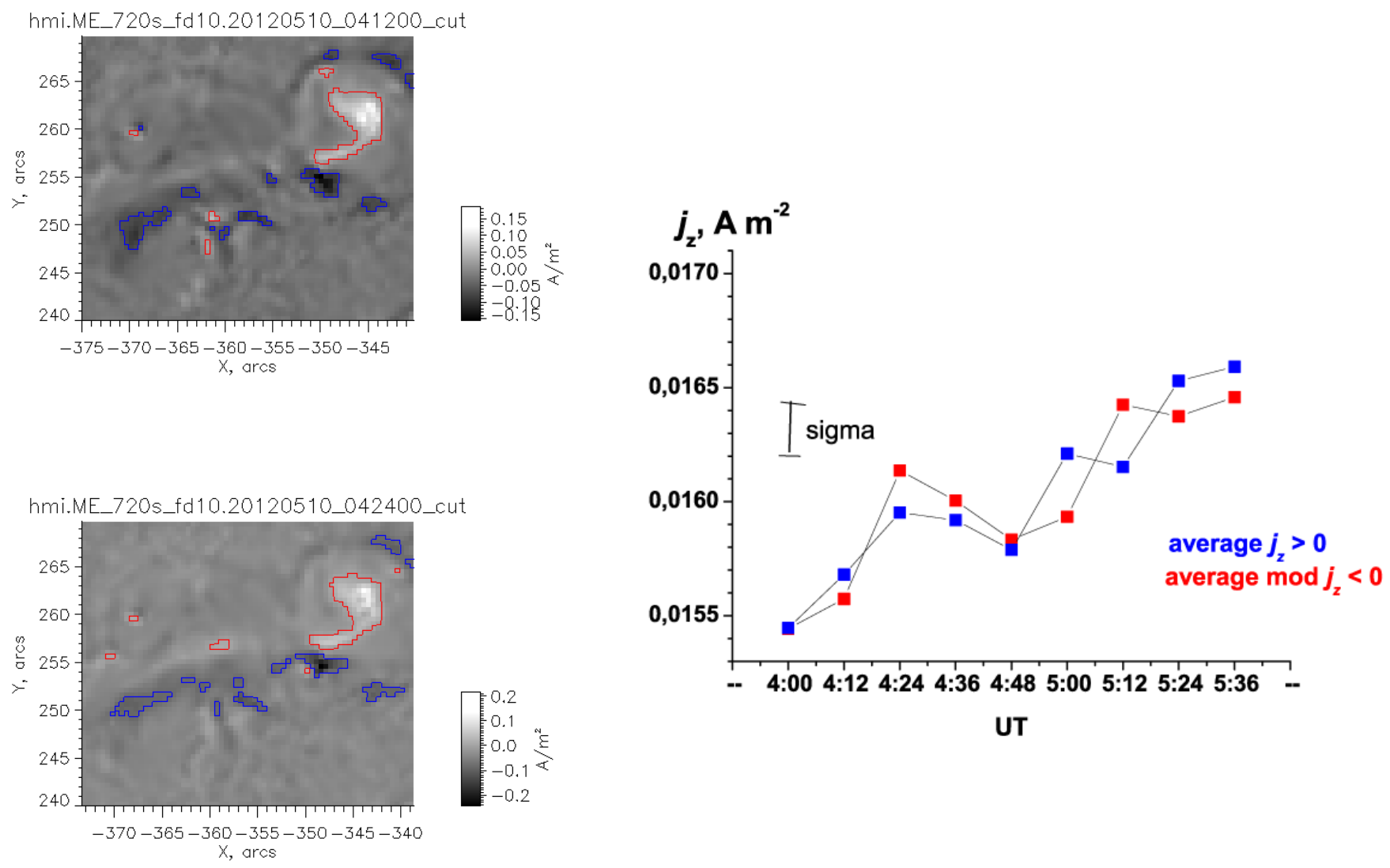

Figure 6. Left: (the background) fragments of the maps of vertical currents, $j_{z}$, calculated for 04:12 UT and 04:24 UT, i.e., before and after the flare maximum, respectively; (the contours) levels $j_{z} \pm 0.05 \mathrm{~A} / \mathrm{m}^{2}$ for $j_{z}<0$ (red color) and $j_{z}>0$ (blue color). The scales show the distance from the disk center in arcsec. Right: evolution of the mean current $j_{z}>0$ and $j_{z}<0$ on May 10 , 2012. For the magnetic field of negative sign, the modulus of $j_{z}$ is shown. The scale provides the time in the form h:mm (UT). 


\section{Evidence of the Relationship between the Emerging Magnetic Fields, Electric Currents, and Solar Flares Observed on May 10, 2012 - 12/16}

This M1.7 flare was also rather hard. The FERMI space mission recorded the emission in the range of about $100 \mathrm{keV}$. At the same time (about 20:24 UT), significant radiation fluxes in the energy range above $50 \mathrm{keV}$ with a maximum of more than 2000 pulses per second were recorded by the Suzaku spacecraft (see the catalogue Suzaku Wide-band All-sky Monitor (WAM): http://www.astro.isas.jaxa.jp/suzaku/HXD-WAM/WAM-GRB/solar/untrig/120510202227.html). Thus, the conditions that lead to the events of the type of a "fast" sigmoid can arise in AR with a period from several hours to a day.

Most of the flares of class $\mathrm{C}$ on the day under discussion had a different origin than the M-flares. Let us consider by way of example the flare T7.9, which was observed at the decay of the soft X-ray emission from our principal event. The flare occurred at 05:04 UT over the polarity inversion line between the spots we are considering and the leading spots. Then, the flare nodes extended from this point directly to the large spot. Such weak events might be triggered by the activity in the latter. In some cases, this activity is due to the emergence of a new field in the vicinity of the spot or with its rotation, or simply with the formation of new magnetic hills. Some of the weak events are rather hard, but they occur usually at the decay of the soft X-ray emission from major flares. Their hardness is due to the conditions favorable for particle acceleration in the traps where a certain number of electrons with energies above $10 \mathrm{keV}$ still remain after the previous event (Vybornov et al., 2015).

\section{Results and Discussion}

Our conclusions are as follows:

1) In the event under examination, there actually occurred a pulse-like energy release accompanied by the bursts of hard X-ray and microwave emission. Simultaneously, a response of the photosphere was recorded in the form of an sunquake. There is every reason to believe that at the same time, the formation of a sigmoid flare began, but it was not completed. Similar processes were repeated in the second M-flare at 20:20 UT, but were absent in many weak flares of class C in the other parts of this AR.

2) Full-vector magnetic data were used to calculate the vertical currents throughout the AR. A simple pattern of the current system that consists of the loop currents above the neutral line (with allowance for the shear) and the currents closed under the photosphere does not agree with the results of calculations for first flare. However, if such calculations are carried out in an area confined to the size of the flare sigmoid, we will find local maxima in the time variation of both positive and negative vertical currents. For the longer time interval, the time variations of averaged vertical currents determines the overall evolution of the flare activity in a given AR.

3) This example of moderate power event has demonstrated at both the sunquake and particle acceleration are more effective in sufficiently strong magnetic fields residing near the polarity inversion line.

Let us briefly discuss the connection of our results to the gentral problem of the development of non-stationary processes. The phenomenon of sigmoid flares has been widely discussed in literature. A rich experience of numerical simulation of these processes has been accumulated (e.g., see (Hood et al., 2012, and references therein). Formerly, many authors adhered to the idea of reconnection of magneticfield lines. Now, it is believed that the main factor is the dynamics of the currents, while the role of the reconnection becomes significant at high altitudes in the corona. The earlier conclusion that plasma flux ropes with the current are not ejected directly from the top of the convection zone remains valid. Large-scale sub-photospheric motions shear the feet of the field lines along the polarity inversion line. In addition to that, the helicity is also carried out from sub-photospheric layers.

So, the emergence of the shear and helicity creates conditions for the formation of a magnetic flux 


\section{Evidence of the Relationship between the Emerging Magnetic Fields, Electric Currents, and Solar Flares Observed on May 10, 2012 - 13/16}

rope. Both theoretical reasoning and extrapolation of the nonlinear force-free fields to the corona suggest that the evolution of the AR magnetic field described above must result in accumulation of some amount of free energy in the chromosphere, which can be spent on the development of nonstationary processes.

The very moment of a large impulsive flare is associated with the reconstruction of the current system in AR. Namely, the bunch of force lines and the currents along them change essentially approaching the ideal configuration, i.e., currents along the loops, which are located in projection onto the photosphere at a large angle to the neutral line.

After the flux rope is released, the magnetic configuration restores its original state. If the previously existing large-scale sub-photospheric motions continue, the formation of the flux rope is repeated, i.e., a series of similar flares occur. These considerations agree with the results of the latest numerical calculations (Savcheva, 2016).

One important issue is to figure out a mechanism of the flare. We note that prior a flare the strong current in the magnetic rope has to be closed under the photosphere. This suggests the toroid in the meridian plane, partially risen above the photosphere. This geometry of the twisted magnetic fiekd is given in Figure 2 in Titov and Démoulin (1999).

In fact, the flux ropes can exist for a long time even in active regions of rather complex topology. However, in some cases, especially at geat currents, a torus instability can arise, particularly in the presence of strong currents (Shafranov, 1966). Conditions for the development of such instabilities were determined in laboratory experiments (Myer et al., 2015). Of course, for flares on the Sun and low-mass stars, we cannot insist upon a specific type of instability, and more resonable to consider a more general case of hydrodynamical instabilities in the plasma rope with the strong current.

Simulation of the current system throughout the AR is an important problem. Only now it has become clear that strong currents and large Lorenz forces exist very low (up to 2-3 thousand km above the photosphere). Higher in the corona, the fields rapidly become potential, and thin current sheets are possibly present.

Another evidence in addition to the general considerations, is a complex behavior of the polarization of microwave radiation. It is known that the polarization changes its sign when passing from the disk center to the limb. This is because the beam enters quasi-transverse magnetic field (QT-region) (e.g., see Zheleznyakov, 1963; Peterova, 1973). In our case (see Fig. 3) we see the inversion of the polarization sign at the frequency of $9.4 \mathrm{GHz}$ at the same time (without motion of the source).

In addition to this well-known effect, a secondary short-time inversion was recorded at the frequency of $2 \mathrm{GHz}$ with the maximum polarized emission at 04:16 UT. This, in turn, suggests a complex topology of the coronal magnetic field, when a complex polarization picture is due both to the projection effects, and to increasing complexity of the coronal topology.

Thus, an "ideal" current system can exist within a small volume. But usually it is immersed into various independent magnetic fluxes separated by separatrix surfaces. As a result, the particularities of the polarized emission that appear at low frequencies in the microwave range indicate the role of reconnection in the coronal layers in AR. Note that the foundations for investigation of the topology of the solar corona were laid by Lee et al. (2010).

Note also that two scenarios of events are possible in the Sun: (1) with coronal mass ejection (CME), frequently observed, particularly, in major flares and (2) with a rising loop without ejection (rarer events). The latter was observed in the M-flares on May 10, 2012. 


\section{Evidence of the Relationship between the Emerging Magnetic Fields, Electric Currents, and Solar Flares Observed on May 10, 2012 - 14/16}

\section{Acknowledgments}

We are grateful to the reviewers and to V.I. Vybornov for his assistance in the course of the work. The SDO data are the courtesy of NASA and the HMI and AIA science teams. We acknowledge the use of RHESSI, GOES and Fermi/GBM data. We are grateful to the instrumental teams operating the Nobeyama solar facilities and GOES satellites.

The work was supported by the Russian Foundation for Basic Research, project nos. 14-02-00922, 16-32-00315, 15-32-20504, 15-02-03835, 15-02-01089, 15-02-01077, 16-02-00749, and particularly by Program no. 7 of the Presidium of the Russian Academy of Sciences.

\section{References}

[1] Buitrago-Casas, J. C., Martínez Oliveros, J. C., Lindsey, C., Calvo-Mozo, B., Krucker, S., Glesener, L., Zharkov, S., A Statistical Correlation of Sunquakes Based on Their Seismic and White-Light Emission. Solar Phys., 2015, 290, Issue 11, pp.3151-3162.

[2] Fisher, G. H., Bercik, D. J., Welsch, B. T., Hudson, H. S., Global Forces in Eruptive Solar Flares: The Lorentz Force Acting on the Solar Atmosphere and the Solar Interior. 2012, Solar Phys., 277, 59

[3] Fletcher, L., Dennis, B. R., Hudson, H. S., Krucker, S., Phillips, K., Veronig, A., Battaglia,M., Bone, L., Caspi, A., Chen, Q., Gallagher, P., Grigis, P. T., Ji, H., Liu, W.,Milligan, R. O., and Temmer, M., An Observational Overview of Solar Flares, Space Sci. Rev. 2011, 159, 19.

[4] Grigor'eva, I.Yu., Livshits, M.A., Rudenko, G.V., and Mysh'yakov, I.I., Active regions near the recent solar cycle minimum: Relation between plasma heating and electrical current. Astron. Rep., 2013, 57, no. 8, pp. 611-621.

[5] Hood, A. W.; Archontis, V.; MacTaggart, D.3D MHD Flux Emergence Experiments: Idealised Models and Coronal Interactions. Sol. Phys., 2012, 278, Issue 1, pp.3-31.

[6] Kostyuk, N.D., Pickelner, S.B. Gasdynamics of a flare region heated by a stream of high-velocity electrons. Astron. Zh., 1974, 51, p.1002-1016 (Soviet Astronomy, 18, 1975, p. 590-599)

[7] Krucker, S.; Battaglia, M.; Cargill, P. J.; Fletcher, L.; Hudson, H. S.; MacKinnon, A. L.; Masuda, S.; Sui, L.; Tomczak, M.; Veronig, A. L.; Vlahos, L.; White, S. M.,Hard X-ray emission from the solar corona. Astron. Astrophys. Rev., 2008, 16, p. 155-208.

[8] Lee, J.-Y.; Barnes, Graham; Leka, K. D.; Reeves, Katharine K.; Korreck, K. E.; Golub, L.; DeLuca, E. E., The Role of Magnetic Topology in the Heating of Active Region Coronal Loops, Astrophys. J., 2010, 723, Issue 2, pp. 1493-1506.

[9] Lemen, J.R., Title, A.M., Akin, D.J., Boerner, P.F., Chou, C., Drake, J.F., Duncan, D.W.,Edwards, C.G., Friedlaender, F.M., Heyman, G.F., Hurlburt, N.E., Katz, N.L., Kushner,G.D., Levay, M., Lindgren, R.W., Mathur, D.P., McFeaters, E.L., Mitchell, S., Rehse, R.A., Schrijver, C.J., Springer, L.A., Stern, R.A., Tarbell, T.D., Wuelser, J.-P., Wolfson, C.J., Yanari, C., Bookbinder, J.A., Cheimets, P.N., Caldwell, D., Deluca, E.E., Gates, R., Golub, L., Park, S., Podgorski, W.A., Bush, R.I., Scherrer, P.H., Gummin, M.A., Smith, P., Auker, G., Jerram, P., Pool, P., Soufli, R., Windt, D.L., Beardsley, S., Clapp, M., Lang, J., Waltham, N., Solar Phys. 275, 17 (2012).

[10] Matyukhin, Y. G.; Tomozov, V. M., Quasistatic Evolution of Magnetic Structures on the Sun and Their Topological Reorganization. Soviet Astronomy, 1991, 35, No. 1, P. 79. 


\section{Evidence of the Relationship between the Emerging Magnetic Fields, Electric Currents, and Solar Flares Observed on May 10, 2012 - 15/16}

[11] Lin, R.P., Dennis, B.R., Hurford, G.J., Smith, D.M., Zehnder, A., Harvey, P.R., Curtis, D.W., Pankow, D., Turin, P., Bester, M., Csillaghy, A., Lewis, M., Madden, N., van Beek, H.F., Appleby, M., Raudorf, T., McTiernan, J., Ramaty, R., Schmahl, E., Schwartz, R., Krucker, S., Abiad, R., Quinn, T., Berg, P., Hashii, M., Sterling, R., Jackson, R., Pratt, R., Campbell,R.D., Malone, D., Landis, D., Barrington-Leigh, C.P., Slassi-Sennou, S., Cork, C., Clark, D., Amato, D., Orwig, L., Boyle, R., Banks, I.S., Shirey, K., Tolbert, A.K., Zarro, D., Snow, F., Thomsen, K., Henneck, R., McHedlishvili, A., Ming, P., Fivian, M., Jordan, J., Wanner, R., Crubb, J., Preble, J., Matranga, M., Benz, A., Hudson, H., Canfield, R.C., Holman, G.D., Crannell, C., Kosugi, T., Emslie, A.G., Vilmer, N., Brown, J.C., Johns-Krull, C., Aschwanden, M., Metcalf, T., Conway, A.: 2002, The Reuven Ramaty HighEnergy Solar Spectroscopic Imager (RHESSI). Solar Phys. 210, 3. DOI. ADS.

[12] Metcalf, Thomas R.; De Rosa, Marc L.; Schrijver, Carolus J.; Barnes, Graham; van Ballegooijen, Adriaan A.; Wiegelmann, Thomas; Wheatland, Michael S.; Valori, Gherardo; McTtiernan, James M., Nonlinear Force-Free Modeling of Coronal Magnetic Fields. II. Modeling a Filament Arcade and Simulated Chromospheric and Photospheric Vector Fields. Solar Phys., 2008, 247, Issue 2, pp.269299.

[13] Myers, Clayton E.; Yamada, Masaaki; Ji, Hantao; Yoo, Jongsoo; Fox, William; Jara-Almonte, Jonathan; Savcheva, Antonia; Deluca, Edward E., A dynamic magnetic tension force as the cause of failed solar eruptions, Nature, 2015, 528, Issue 7583, pp. 526-529.

[14] Nakajima, H., Sekiguchi, H., Sawa, M., Kai, K., Kawashima, S., The radiometer and polarimeters at 80, 35, and $17 \mathrm{GHz}$ for solar observations at Nobeyama. Pub. Astron. Soc. Japan, 1985, 37, 163.

[15] Nakajima, H., Nishio, M., Enome, S., Shibasaki, K., Takano, T., Hanaoka, Y., Torii, C.,Sekiguchi, H., Bushimata, T., Kawashima, S., Shinohara, N.,Irimajiri, Y., Koshiishi, H., Kosugi, T., Shiomi, Y., Sawa, M., Kai, K., The Nobeyama radioheliograph. IEEE Proceedings, 1994, 82, 705.

[16] Peterova,N.G., Akhmedov, Sh.B. Astron. zhurn. 50, 1220 (1973).

[17] Prist, E., Forbs, T., Magnetic Reconnection, Cambridge Univ. Press, 2000.

[18] Rust, David M., Magnetic Field Changes during Solar Flares. Astron. Journal, 1968, 73, p. 74.

[19] Rudenko, G.V., Myshyakov, I.I., Solar Phys. 2009, 257, 287.

[20] Savcheva, A.; Pariat, E.; McKillop, S.; McCauley, P.; Hanson, E.; Su, Y.; DeLuca, E. E., The Relation between Solar Eruption Topologies and Observed Flare Features. II. Dynamical Evolution.The Astrophys. Journal, 2016, 817Volume 817, Issue 1, article id. 43, 22 pp.

[21] Schou, J., Scherrer, P.H., Bush, R.I., Wachter, R., Couvidat, S., Rabello-Soares, M.C., Bogart,R.S., Hoeksema, J.T., Liu, Y., Duvall, T.L., Akin, D.J., Allard, B.A., Miles, J.W., Rairden,R., Shine, R.A., Tarbell, T.D., Title, A.M., Wolfson, C.J., Elmore, D.F., Norton, A.A.,Tomczyk, S., Design and Ground Calibration of the Helioseismic and Magnetic Imager(HMI) Instrument on the Solar Dynamics Observatory (SDO). Solar Phys., 2012, 275, 229.

[22] Shafranov, V. Plasma equilibrium in a magnetic field. Rev. Plasma Phys. (ed. Leontovich, M. A.), 2, 103-152 (1966).

[23] Sharykin, I. N.; Kosovichev, A. G., Dynamics of Electric Currents, Magnetic Field Topology, and Helioseismic Response of a Solar Flare. Astrophys. J., 2015, 808, Issue 1, article id. 72, 9 pp.

[24] Sharykin, I. N.; Kosovichev, A. G.; Zimovets, I. V., Energy Release and Initiation of a Sunquake in a C-class Flare. The Astrophys. J., 2015, 807, Issue 1, article id. 102, 9 pp. 


\section{Evidence of the Relationship between the Emerging Magnetic Fields, Electric Currents, and Solar Flares Observed on May 10, 2012 - 16/16}

[25] Shibasaki, K., Ishiguro, M., Enome, S., Solar Radio Acquisition and Communication System /SORDACS/ of Toyokawa Observatory. Proceedings of the Research Institute of Atmospherics, 1979, Nagoya University 26, 117. ADS.

[26] Severny A.B. Some problems of the physics of the Sun. Moscow, Nauka, Fiz.-mat.lit., 1988, 224 p.

[27] Titov, V. S.; Démoulin, P. Basic topology of twisted magnetic configurations in solar flares. Astron. Astrophys., 1999, 351, p.707-720.

[28] V. I. Vybornov, I. Yu. Grigor'eva, M. A. Livshits, and E. F. Ivanov. 2015, Emergence of Hard X-Rays at Weak Nonstationary Processes in Active Regions. Geomagnetizm and Aeronomy, 55, , No. 8, pp. 1112-1117.

[29] Wheatland, M. S.; Sturrock, P. A.; Roumeliotis, G., An Optimization Approach to Reconstructing Force-free Fields. The Astrophys. J., 2000, 540, Issue 2, pp. 1150-1155.

[30] Zaitsev, V. V.; Stepanov, A. V., Coronal magnetic loops. Uspekhi Fizicheskikh Nauk, 2008, 178 , No. 11, p. 1165-1204.

[31] V.V. Zhelezniakov, E.Ia. Zlotneyk, Astron. zhurn. 40, 633, (1963). 